\title{
High folate status is positively associated with cognitive function, irrespective of B12 status. Findings from The Irish Longitudinal Study on Ageing (TILDA)
}

\author{
Deirdre O’Connor ${ }^{1}$, Eamon Laird ${ }^{1}$, Daniel Carey ${ }^{1}$, Aisling O’Halloran ${ }^{1}$, \\ Rose Anne Kenny ${ }^{1}$ and Anne M. Molloy ${ }^{2}$ \\ ${ }^{1}$ The Irish Longitudinal Study on Ageing (TILDA), Trinity College Dublin, Dublin 2, Ireland and ${ }^{2}$ School of \\ Medicine, Trinity College Dublin, Dublin 2, Ireland.
}

Recent data suggest that an imbalance of low vitamin $\mathrm{B}_{12}$ (cobalamin) and high folate status may be associated with negative health outcomes in older adults and children ${ }^{(1)}$. Moreover, it has been previously shown that high folate status or the use of folic acid supplements may have a negative effect on cognitive function in older people with diminished $\mathrm{B}_{12}$ status ${ }^{(2)}$. However, to date, other than the Mini-Mental State Examination (MMSE), few studies have examined the interaction of low $\mathrm{B}_{12}$ and high folate status and individual cognitive domains.

The aim of this study was to establish the prevalence of four $\mathrm{B}_{12}$ and folate profiles in the older adult population in Ireland and to examine the relationship with different measures of cognitive function using data from Wave 1 (2009-2011) of TILDA, a nationally representative cohort of community-dwelling adults aged 50 and over $(n=8,175)^{(3-5)}$.

Blood samples were analysed for concentrations of plasma $B_{12}$ and folate $(n=5,035)$. In this study, the four profiles were defined as: Group 1 'normal B12/normal folate' (total cobalamin $>258 \mathrm{pmol} / \mathrm{L}$, folate $\leq 45 \cdot 3 \mathrm{nmol} / \mathrm{L}$ ), Group 2 'normal B12/high folate' (total cobalamin $>258 \mathrm{pmol} / \mathrm{L}$, folate $>45.3 \mathrm{nmol} / \mathrm{L}$ ), Group 3 'low B12/high folate' (total cobalamin $<=258 \mathrm{pmol} / \mathrm{L}$, folate $>45 \cdot 3 \mathrm{nmol} / \mathrm{L}$ ), and Group 4 'low B12/normal folate' (total cobalamin $\leq 258 \mathrm{pmol} / \mathrm{L}$, folate $\leq 45 \cdot 3 \mathrm{nmol} / \mathrm{L}$ ). The cognitive battery included tests of global cognition ((MMSE) and the Montreal Cognitive Assessment (MoCA)), executive function (Verbal Fluency, Visual Reasoning and Colour Trails Test 2 (CTT2)), memory (Picture Recognition and Recall, and a 10-word Immediate and Delayed Recall test), attention (Colour Trails Test 1 (CTT1) and the Sustained Attention to Response Task (SART)) and speed of processing (Choice Reaction Time (CRT)). Multiple regression analyses were used to examine relationships of each $\mathrm{B}_{12}$ and folate profile with these measures of cognitive function, controlling for known covariates.

The mean (SD) age of the population was $63 \cdot 2$ years (9.8), 51.1\% were female. The prevalence of Group 1 was $61 \cdot 2 \%$ [60.0 to 62.8] ([95\% CI]), Group 2 was 8.0\% [7.0 to 9.0]; Group 3 was $1.7 \%$ [1.3 to 2.2] and Group 4 was 29.1\% [27.7 to 31.0]. Relative to Group 1, Group 2 was associated with an increase in MMSE scores $(0.83$ [0.72 to 0.95]) (IRR [95\% CI]; $P=0.007)$, whilst for the MoCA, Group $2(0.91$ [0.84-0.97]) (IRR [95\% CI]; $P=0.007)$ and Group $3(0.88$ [0.79 to 0.97]) (IRR [95\% CI]; $P=0.009)$ were associated with increased scores. Group $3(-0.08[-0.14$ to -0.01$])$ (coefficient; $[95 \% \mathrm{CI}] ; P=0.02)$ and Group $4(-0.03[-0.05$ to -0.1$] ; P$ $=0.01)$ were associated with better CTT2 scores and Group 3 was also positively associated with Picture Recognition (1.04 [1.01 to 1.07]) (IRR [95\% CI]; $P=0.003$ ). Group 3 and Group 4 were positively associated with Delayed Recall (0.84 [0.23 to 1.40]) (coefficient; $[95 \% \mathrm{CI}] ; P=0.003$ and 0.20 [0.05 to 0.34$]$ (coefficient; $[95 \% \mathrm{CI}] ; P=0.008$, respectively). The SART was positively associated with Group $2(0.85[0.75$ to $0 \cdot 96])$ (IRR $[95 \% \mathrm{CI}] ; P=0.01)$.

Previous evidence has suggested that low $\mathrm{B}_{12}$ status, coupled with high folate status may be associated with a decline in cognitive function. Our data do not support this hypothesis. Contrarily, our data suggest that in several domains of cognitive function, including global cognition, shigh folate status is associated with improved scores, irrespective of $\mathrm{B}_{12}$ status, and thus potentially has a protective effect. These findings may have significant implications for future policy recommendations in Ireland, where a voluntary folic acid fortification programme is currently in place.

1. Paul L \& Selhub J (2017) Mol Aspects Med 53, 43-7.

2. Moore EM, Ames D, Mander AG et al. (2014) J Alzheimers Dis 39, 661-8.

3. Cronin H, O'Regan C, Finucane C et al. (2013) J Am Geriatr Soc 61, (s2).

4. Whelan BJ \& Savva GM (2013) J Am Geriatr Soc 2 61, (s2).

5. Savva GM, Donoghue OA, Horgan F et al. (2013) J Gerontol A Biol Sci Med Sci 68, 441-6. 